\title{
Early Dementia Diagnosis Based on DNN Based Correlational Analysis and Fisher Criterion Based LDA using Morphological Brain Multiplexes
}

\author{
Ambili A.V., A. V. Senthil Kumar, Amit Dutta
}

\begin{abstract}
The expanding recurrence of dementia happening is a disturbing patterning that has incited dire research intending to avert the improvement of the sickness. Diagnosing dementia in its beginning periods is an urgent advance in averting the improvement of the ailment into exacerbated side effects. Early mild cognitive impairment (EMCI) is the early symptom of dementia. This can be analyzed using mapping mind associations utilizing Magnetic Resonance Imaging (MRI). In the approach, for improving the correlational block, we presented an enhanced classifier also, for improving the performance of discriminative block, an optimized LDA is to be proposed. For correlational analysis, Deep Neural Network (DNN) is presented in this work. Besides, for discriminative analysis, a novel and efficient feature selection method is presented. Fisher criterion is used to select the most discriminatory and appropriate features to ensure consistent feature selection and classifier learning goals and to improve the classifier's performance. In the Mat lab framework this proposed method is implemented. The performance of this proposed approach is evaluated concerning Accuracy, Sensitivity, and Specificity.
\end{abstract}

Keywords: Brain multiplex, Correlative analysis, Dementia, discriminant analysis DNN, Fisher criterion

\section{INTRODUCTION}

Dementia is the staggering sicknesses that lack broad consideration from relatives and guardians. Since the side effects of dementia could be very mind-boggling and may change ramblingly, family and guardian experience the ill effects of gloom and high mental worry from patient consideration [1]. In older adults, the symptom of dementia is decreased mobility and increased risk of falls [2]. Dementia with Lewy bodies shares common clinical, neuropsychological and pathological features with Parkinson's disease dementia (PDD) and other dementia subtypes, such as Alzheimer's disease (AD) [3]. While Alzheimer's disease is the most typical organic reason behind dementia, there are several different causes, like tube disorders, e.g., strokes, brain tumors, traumatic brain injuries, or front-temporal lobe degeneration [4].

Revised Manuscript Received on December 30, 2019.

* Correspondence Author

Ambili. A.V, Research Scholar, PG Research and Computer Application, Hindustan College of Arts and Science, Coimbatore. India.

A.V. Senthil Kumar, Director, PG Research and Computer Application, Hindustan College of Arts and Science, Coimbatore. India.

Amit Dutta, Deputy Director, All India Council for Technical Education New Delhi, India.

(c) The Authors. Published by Blue Eyes Intelligence Engineering and Sciences Publication (BEIESP). This is an open access article under the CC BY-NC-ND license (http://creativecommons.org/licenses/by-nc-nd/4.0/)
Structural and functional magnetic resonance imaging (MRI) has been extensively applied to identify brain abnormalities in MCI patients, to perform early diagnosis and estimate time to progression to $\mathrm{AD}$ or other Dementias [5]. It may be challenging to identify early cognitive impairment (EMCI) when mapping brain connections using a disordered brain MRI. An MRI uses the phenomena of nuclear magnetic resonance i.e. it uses magnetic and radio waves, thus there is no risk of exposure to X-rays or any other damages of radiation and a detailed internal picture of the human body can be obtained [6]. In diagnosing patients with cognitive disorders, such as memory problems, to remove any possible causes of symptoms, anatomic MRI is regularly conducted in clinical practice. Consequently, MRIbased atrophy biomarkers have minimal cost impact because MRI scanning is often part of the standard evaluation [7]. MRI techniques mainly used for Morphological brain networks. We tend to propose a cooperative correlation and discriminative learning system, pairing first brain multiplexes, each created using a different set of MBNs. More specifically, Morphological brain networks are designed to be incorporated into a morphological network structure consisting of intralayer and interlayer networks [8].

For early dementia diagnosis, Rory Raeper, Anna Lisowska and Islem Rekik [9] have displayed Cooperative Correlation and Discriminative ensemble classifier learning using morphological brain multiplexes. Both correlational and discriminative strategies have a huge divergence in their hemispheric display, with the right hemispheres yielding better results reliably. The reference CCA approach on the left side of the equator performs especially ineffectively and discernibly damages the presentation of the integrated technique. For the most part, the left hemisphere contains highlights which may be more enthusiastically to distinguish, with dementia perhaps showing in essentially various ways crosswise over sides of the equator. Furthermore, the discriminative strategies perform inadequately on the left side of the equator, with losing a significant accuracy. Along these lines, to further improve the aftereffects of the left half of the globe would be a reasonable beginning heading for future work. For improving the correlational square, an upgraded classifier is to be exhibited. Additionally, for developing the exhibition of the discriminative square, an upgraded LDA is to be introduced. Contributions of this proposed approach are described as follows:For correlational analysis, Deep Neural Network (DNN) is presented in this approach. Besides, for discriminative analysis, a novel and efficient feature selection method is presented. 
Fisher criterion is used to select the most discriminative and appropriate features so that the objectives of feature selection and classifier learning are consistent and the performance of the classifier is to be improved. This proposed approach is implemented in the platform Matlab. The performance of this proposed approach is evaluated in terms of Accuracy, Sensitivity, and Specificity. The remaining part of this paper is represented as follows. Section 2 discusses some recent literature that centered on analysis on early dementia diagnosis. Segment 3 proposes a DNN primarily based correlational analysis and Fisher Separation Criterion based LDA mistreatment morphological brain multiplexes. The results of this projected approach are mentioned in section 4 . Finally, the conclusion of this analysis work is represented in segment 5 .

\section{RELATED WORK}

Kawanish K Kawanish K., et al. [10] had mentioned an unsupervised anomaly detection technique using healthy information solely and additionally aim to propose a system that provides the chance of being dementedness (or different sicknesses) supported the variations from the information of healthy cases. From the point of view for early detection of dementedness, the most drawbacks are that there is a regular glance at the weakness of research material and also the question of making a dementedness sample. To solve these issues, the researchers are specialized in drawing experiments using a pill terminal to build a demented detection system; this can be adapted to change the quality of the drawing together with the digits, characters, and images for increasing dementedness screening opportunities. Chen, D., et al. [11] had illustrated Module variations of glucose metabolic brain network among Alzheimer's (AD), Parkinson's disease dementedness (PDD), Lewy body dementedness (DLB) and health management. The graphtheoretical brain network analysis technique is taken into account as a preferred and universal technique within the field of the neurosciences. It will facilitate neuroscientists to research the neuropathology of dementedness, particularly to know dementedness subtypes. As a result, findings during this paper square measure expected to be helpful for neuroscientists to additional perceive the pathology of $\mathrm{AD}$, PDD, and DLB.

Bharanidharan, $\mathrm{N}$, and Rajaguru, $\mathrm{H}$, et al. [12] projected to uses the Harmony Search optimization technique to classify dementedness through magnetic resonance imaging pictures. Harmony Search algorithmic program is employed extensively for the optimization downside, feature choice and training Neural Networks. However, mistreatment Harmony looks for the classification of medical pictures is ingenious. Soft computing techniques may be utilized in the machine-controlled classification of dementedness, to assist the practitioner in dementedness diagnosing. Kobayashi, T., et al. [13] developed dementedness diagnosing System supported the natural oral communication between AN older person and a golem. this technique consists of the perform to come up with a situation from the Hasegawa dementedness rating scale-revised (HDS-R). We tend to confirm the effectiveness of technique by analysis experiment in examination with the standard HDS-R method operated by examiners. Grande, G. Et al.[14] proposed a gait rate test to improve the diagnosis of prodromic madness. They tend to seek to review the evidence relating to the correlation of slow gait speed with mental regression and madness and to address the possible mutual mechanisms resulting in cognitive characteristics and motor dysfunction, under the unifying theory that body and mind are intimately connected. The interaction of body and mind appears relevant throughout the event of psychological feature decline and insanity. The mensuration of gait speed could improve the detection of prodromic insanity and psychological feature impairment in people with and while not initial psychological feature deficits. Schrum, M., et al. [15] projected that a golem medical care mechanism is a good tool for encouraging exercise in insanity patients. Such a mechanism can facilitate address issues like the value of care and a shortage of health care employees. The center rate is employed as feedback to determine that exercise move ought to be incontestable. The results we've got found are promising and that we hope to continue this work via future studies.

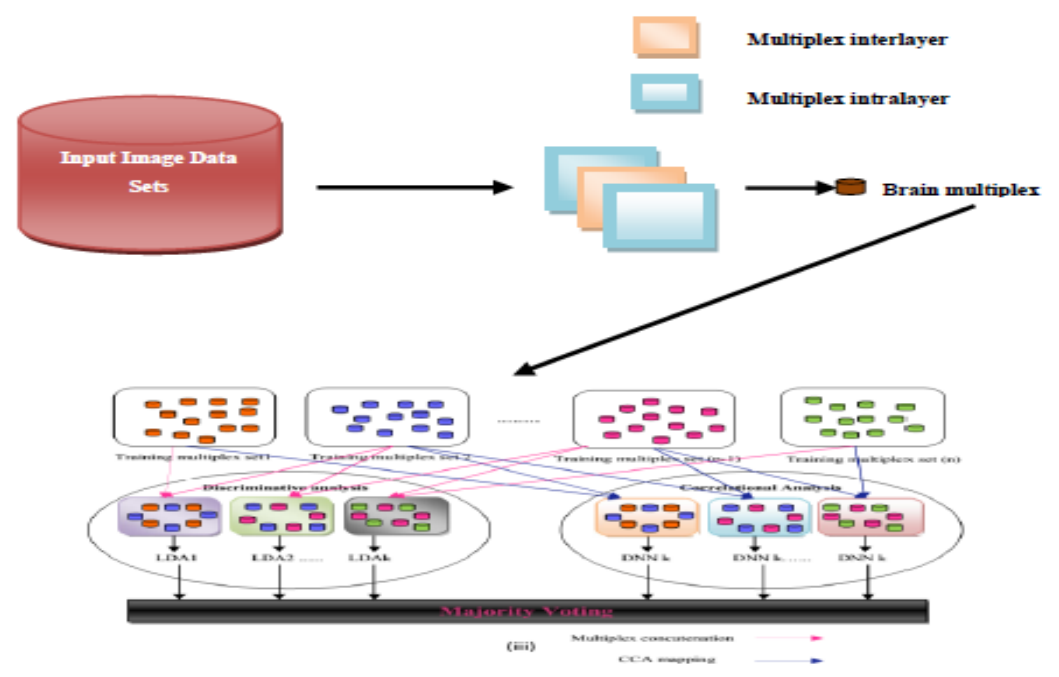

Fig.1- Proposed correlational and discriminative ensemble learning using brain multiplexes (Paired LDA discriminative and DNN correlational- morphological brain multiplexes). 


\section{PROPOSED METHOD}

In this work, to upgrade the correlational square, we exhibited an improved classifier additionally, for improving the presentation of the discriminative block, an optimized LDA is to be proposed. An enhanced Linear Discriminative Analysis (LDA) and its variations distinguish brain features that discriminate two brain states. The target of the proposed feature selection strategy is to some degree predictable with the LDA (both pursue the Fisher standard) and hence the combination of the proposed feature selection technique and LDA is relied upon to accomplish higher arrangement accuracy. For the Correlation investigation, Deep Neural Network (DNN) is proposed in this work. DNN incorporates two stages: pre-preparing and tweaking stages in its parameter learning. The preparation procedure is accelerating with the guide of the LDA optimized algorithm. The significant objective of the correlational investigation is to recognize profoundly connected features inside the information, choosing a subset of features from the first information to expel excess which may the prediction accuracy. Also, for discriminative examination, a novel and proficient element determination technique are exhibited. Fisher measure is utilized to choose the most discriminative and proper features with the goal that the targets of feature choice and classifier learning are predictable and the exhibition of the classifier is to be improved. This proposed methodology is actualized in the foundation of Matlab. The exhibition of this proposed correlational and discriminative outfitting the hang of utilizing brain multiplexes is shown in fig.1. For every conceivable mixture of multiplex combines, each pair is passed into the cluster structure, comprising of correlational learning blocks that are mapped by DNN and discriminative blocks are mapped and isolated into two classes by LDA. The two blocks turn out to be anticipated names of categories to look at passionate subjects about investigating consequent multiplex sets. The last class imprint is assigned through the majority possibility on names allotted by the two blocks.

\section{A. Morphological Brain multiplexes}

In a nonexclusive manner, we characterize a brain multiplex $\mathrm{M}^{\mathrm{x}}$ utilizing a set of $\mathrm{M}^{\mathrm{n}}$. Inter layers $\mathrm{I}_{1} \ldots \mathrm{I}_{\mathrm{mx}}$ each speak to a solitary perspective on the mind morphology, where between two back to back intra- layers $I_{i}$ and $I_{j}$ we slide an interlayer $L_{i, j}$. This respects the accompanying general multiplex architecture,

$$
\mathrm{M}^{\mathrm{x}}=\left(\mathrm{I}_{1}, \mathrm{~L}_{12}, \mathrm{I}_{2} \ldots \ldots, \mathrm{I}_{\mathrm{j}}, \mathrm{L}_{\mathrm{ij}}, \mathrm{I}_{\mathrm{j}}, \ldots \ldots, \mathrm{I}_{\mathrm{mx}}\right)
$$

Every interlayer is characterized by convolving two back to back intralayers. Every element inline $\mathrm{R}$ and section $\mathrm{C}$ is given beneath, $L_{i, j(R, C)}=\sum_{s} \sum_{t} I_{i}(s, t) I_{j}(R-s+1, C-t+1)$ (2)

We note that for a particular multiplex, we are permitted to investigate likenesses between continuous layers. Henceforth to investigate the connection between every single imaginable blend of intra-layers, we create for each subject " $n$ " multiplexes through just re-requesting the intra- layers systems, in this way producing troupe multiplexes is appeared in underneath

$$
\mathrm{M}=\mathrm{M}^{1} \ldots \ldots . . \mathrm{M}^{\mathrm{n}}
$$

Hence the morphological brain connectivity metrics are symmetric.

\section{B. Discriminative Analysis Using Fisher criterion- based LDA:}

LDA seeks to develop a model that reduces sample separation from the same class and increases sample separability from different classes. The Discriminative method aims to tracking the original features, unlike LDA which projects the features. Using correlation data, Linear Discriminant Analysis (LDA) achieves the highest accuracy. Discriminatory techniques, such as feature selection methods and linear discriminative analysis (LDA) and their variations, define brain features that discriminate against two brain states. By using the fisher criteria, we pick the features of each morphological brain network. For each system of size is $\mathrm{N}^{*} \times \mathrm{N}^{*}$. The extraction of feature vector size is shown in below equation (4).

$$
\left\{\mathrm{N}^{*} \times(\mathrm{N}-1) / 2\right\}
$$

A brain multiplex brought about numerous exceptionally connected features utilized for the gathering realizing, which may to some degree mislead classifier learning. To limit the connection between's various multiplexes when blending them for outfits classifier preparing, we structure a fisher paradigm is appeared in underneath condition,

$$
Z=\frac{\left|v_{p} c_{s} v_{p}\right|}{\left|v_{p} c_{s i} v_{p}\right|}
$$

The arrangement $v_{p}$ can be accomplished by taking care of the summed up Eigen issues of $\mathrm{c}_{\mathrm{s}} \mathrm{v}_{\mathrm{p}}=\lambda \mathrm{c}_{\mathrm{si}} \mathrm{V}_{\mathrm{p}}$ with its driving eigenvalues. $C_{s}$ and $C_{s i}$ are the class scatter and within a class scatter is given below, $c_{s}=\frac{1}{n} \sum_{i=1}^{z} n_{i}\left(m^{v}-m_{o}^{v}\right)\left(m^{v}-m_{o}^{v}\right)^{*}$

$c_{s i}=\frac{1}{n} \sum_{i=1}^{z} \sum_{j=1}^{n i}\left(o_{j}^{i}-m^{v}\right)\left(o_{j}^{i}-m^{v}\right) *$ (7) Where $n=\sum_{i}^{z} n_{i}$ is the total number of samples; $\mathrm{z}$ is the number of classes; $m^{v}=\frac{1}{n_{i}} \sum_{i}^{n_{i}} o_{i}$ is the mean vector of class $i$ and $m_{o}^{v}=\frac{1}{n} \sum_{i}^{x} \sum_{j=1}^{n_{i}} o_{j}^{i}$ is the mean vector over the whole sample set. Consider the feature selection problem, suppose the original feature set is,

$$
\mathrm{f}^{0}=\mathrm{f}_{1}{ }^{0}, \mathrm{f}_{2}{ }^{0}, \ldots \ldots, \mathrm{f}_{\mathrm{n}}^{0}
$$

Where, $\mathrm{n}$ is the dimension of original feature set, the use of feature is to select $\mathrm{x}$ features,

$\mathrm{f}^{\mathrm{x}}=\mathrm{f}_{\mathrm{w}(1)}, \mathrm{f}_{\mathrm{w}(2)}, \ldots \ldots \ldots, \mathrm{f}_{\mathrm{w}(\mathrm{x})}$

From $\mathrm{n}$ unique features which have the biggest component esteem. Here, $w(i)$ is the $\mathrm{i}^{\text {th }}$ feature file in the chose feature subset. 
Denoting the class scatter and inside the class scatter figured dependent on the chose list of capabilities as, and the fisher measure as, $\mathrm{Z}\left(\mathrm{f}^{\mathrm{x}}\right)$ The goal of choosing $\mathrm{x}$ features dependent on the fisher basis can be composed as,

$$
\mathrm{f}^{\mathrm{x}}=\arg \max \mathrm{Z}\left(\mathrm{f}^{\mathrm{x}}\right)
$$

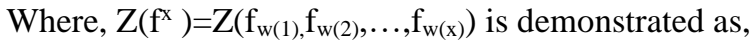

$$
Z\left(f^{x}\right)=\frac{\left|v^{P^{*}} C_{s}\left(f^{x}\right) v^{p}\right|}{\left|v^{P^{*}} C_{s i}\left(f^{x}\right) v^{P}\right|}
$$

Straightforwardly choosing $\mathrm{x}$ features from $\mathrm{n}$ features clients $(\mathrm{x}<<\mathrm{n})$ is an NP-difficult issue. A problematic path is to consecutively choose the highlights in a greedily. Assume we have chosen $q$ features at the following stage. We select the $(\mathrm{q}+1)^{\text {th }}$ feature that boosts the fisher paradigm as, $\mathrm{f}_{(\mathrm{q}+1)}=\arg \max \mathrm{Z}\left(\mathrm{f}_{\mathrm{q}}, \mathrm{f}\right)$

The Fisher criterion improvement value is given below,

$$
\Delta \mathrm{Z}\left(\mathrm{f}_{\mathrm{c}} / \mathrm{f}_{\mathrm{s}}\right)=\mathrm{Z}\left(\mathrm{f}_{\mathrm{c}}, \mathrm{f}_{\mathrm{s}}\right)-\mathrm{Z}\left(\mathrm{f}_{\mathrm{s}}\right)
$$

Where, $\Delta f_{\text {ish }}\left(f_{c} \mid f_{s}\right)$ is spoken to as fisher paradigm improvement esteem, $f_{c}$ is the client's features and $f_{s}$ is chosen a feature. The feature whose negligible Fisher partition improvement esteem in the chose list of capabilities is the biggest among all applicants is favored as another one. Other than the Fisher division foundation, we likewise trust the new chosen highlight is a decent reciprocal one to the current ones. That is, the redundancies among the chose features are relied upon to be little. At long last, we acquired an element choice paradigm is,

$\mathrm{f}_{(\mathrm{q}+1)}=\operatorname{argmax}\left\{\min (\mathrm{fc} / \mathrm{fs})-\lambda \max \ell\left(\mathrm{f}_{\mathrm{c}} . \mathrm{f}_{\mathrm{s}}\right)\right\}$

Where, $f_{q+1}$ is represented as $(\mathrm{q}+1)$ th feature, The final feature selection criterion (14) consists of two functions, One is the Fisher separation criterion evaluating the discriminant characteristic of features and the other is the correlation term which measures the redundancy of features.

\section{Correlation analysis using deep neural network:}

A deep neural network (DNN) is an artificial neural network (ANN) with multiple layers of hidden units between the layers of input and output. Deep learning methods are highly effective when there is a vast amount of samples available among the training stage. Therefore, the proposed Correlational analysis is defined with DNN based approach. During the training of traditional DNN, the weights of the neurons are refreshed in every cycle until the error amongst output and input is not within tolerance. This is time- consuming. In this work, we use DNN to map pairs of multiplex feature vectors extracted from various sets into a shared subspace representing highly-correlated features. This correlational block minimizes the set-specific noise of multiplexes and reduces the dimensionality of. This correlational block minimizes the set-specific noise of multiplexes and reduces the dimensionality of multiplex data. Since every multiplex $\mathbf{M}^{\mathrm{x}} \in \mathrm{M}$ catches an interesting and complex connection between various mind network sees, one needs to look at all morphological cerebrum multiplexes in the outfit $\mathrm{M}$. This will give us an increasingly all-encompassing comprehension of how unequivocal morphological mind associations can be changed by dementia beginning just as how their verifiable high-request (an association of associations) relationship can be influenced. To utilize all the data accessible from various multiplexes, in the correlational learning square of our structure. DNN incorporates two stages: pre-preparing (using generative deep conviction network) and calibrating stages in its parameter learning.

\section{Training In DNN}

In the training stage, use a Deep Belief Network (DBN) which is a deep design and common feedforward framework in which the input goes from the input layer to the output layer through various hidden layers which are more than two layers. The DBN model enables the framework to make visible inceptions dependent on its hidden units' states, which portrays the framework conviction. Here, we executed the Restricted Boltzmann Machine (RBM) to work out the above issue. The structure of the DNN shows up in figure 2 .

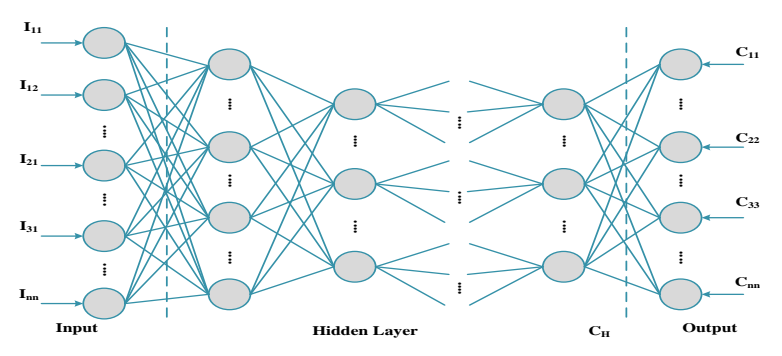

Fig.2 Structure of DNN

\section{Restricted Boltzmann Machine:}

An RBM is a prohibitive sort of Markov random field that has one layer of (ordinarily Bernoulli) stochastic hidden units and one layer of (consistently Bernoulli or Gaussian) stochastic visible or perceptible units. The input of the DNNs is i.e., the vectorization and the output of the network is the decision vector.

Consider ' $R_{m}$ ' be the input data sets where $1 \leq \mathrm{m} \leq \mathrm{M}$ and ' $C$ ' denotes the output data sets. The generalized neural network model can be given as ' $\mathrm{C}$ ' for the whole network output and ' $\mathrm{CH}$ ' for the hidden layer output. As in DNN, there are more hidden layers, the individual element inputs are multiplied by weights in the first hidden layer. Likewise, the individual first hidden element outputs are multiplied by another set of weights in the second hidden layer and so on. The weighted input values are fed to the summing function in the first hidden layer with the neuron bias as in equation (15)

$$
C_{H_{-} 1}(x=1,2 . ., K)=\left(\sum_{m=1}^{M} w_{x m} R_{m}\right)+b_{x}
$$

Where $b_{x}$ constant value serves as a bias, $\mathrm{w}_{\mathrm{xm}}$ the input-tohidden layer interconnection weight of $M$ and $K$ representing the number of inputs and hidden nodes in the first hidden layer.

The activation function which is the output of the first hidden layer is given as

$$
F\left(C_{H_{-1}}(x)\right)=\frac{1}{\left(1+e^{-C_{H_{-1}}(x)}\right)(16)}
$$


Where $\mathrm{F}($.$) is the sigmoid activation function. Therefore,$ the operation of $\mathrm{y}^{\text {th }}$ hidden layer can be generalized as,

$$
C_{H_{-} y}(p)=\left(\sum_{z=1}^{K} w_{p z} F\left(C_{H_{-}(y-1)}(z)\right)\right)+b_{p}
$$

Where $b_{p}$ is the bias of $p^{\text {th }}$ hidden node, $w_{p z}$ is the interconnection weight between the $(\mathrm{y}-1)^{\text {th }}$ hidden layer and $\mathrm{y}^{\text {th }}$ hidden layer with $\mathrm{K}$ hidden nodes.

The activation function which is the output of the $y^{\text {th }}$ hidden layer is given as,

$$
F\left(C_{H_{-} y}(p)\right)=\frac{1}{\left(1+e^{-C_{H_{-}}(p)}\right)}
$$

At the

output layer, the $y^{\text {th }}$ hidden layer output is again multiplied with the interconnection weights (i.e. weight between the $\mathrm{y}^{\text {th }}$ hidden layer and output layer) and then summed up with the bias

$C(q)=F\left(\sum_{p=1}^{K} w_{q p} f\left(C_{H_{-} y}(p)\right)+b_{q}\right)$

Where $\mathrm{w}_{\mathrm{qp}}$ represents the interconnection weight at the $y^{\text {th }}$ hidden layer and output layer having $\mathrm{p}^{\text {th }}$ and $\mathrm{q}^{\text {th }}$ nodes respectively. The activation function at the output layer acts as the output of the whole model. Now the network output is contrasted with the target and difference (i.e. error) is obtained to optimize the network output. The error calculation is as in equation $\varepsilon=\frac{1}{M} \sum_{m=1}^{M}\left(\operatorname{Actual}\left(C_{m}\right)-\operatorname{Pr} \text { edicted }\left(C_{m}\right)\right)^{2}(20)$

Where Predicted $\left(\mathrm{C}_{\mathrm{m}}\right)$ is the estimated network output and Actual (Cm) is the actual output. The error must be minimized for getting optimal network structure. The weight values must, therefore, be adjusted until the error at each iteration reduced. The structure is shown in fig. 3.

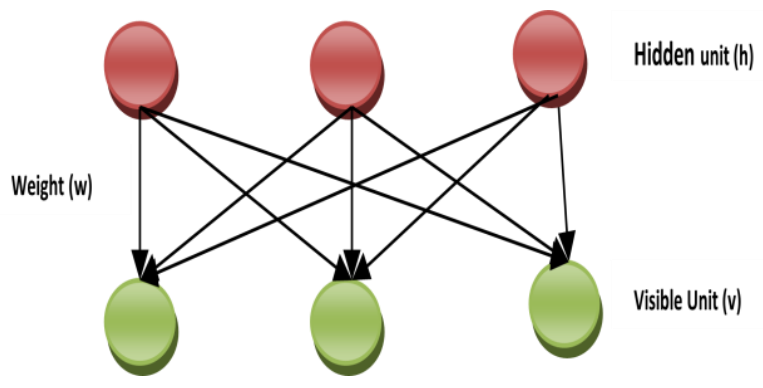

Fig. 3 RBM Structure

\section{Majority Voting}

Finally, we used majority voting to identify the class label of the testing subject, by selecting the highly frequent predicted label outputted by classifiers in both blocks. Highly frequent class labels are obtained from two blocks (i.e.) Discriminative analysis and Correlational analysis using LDA and DNN. The classification accuracy significantly improves when the correlational and discriminative accuracies are comparable independently. However, when the disparity between the correlational and discriminative methods is large, the accuracy generally averages between the two instead of improving. This is probably due to the majority voting being heavily biased towards the lower accuracy, pushing the classifier to select the incorrect label.

\section{$\underline{\text { Steps }}$}

Input: Multiplex feature vectors $\left(\mathrm{R}_{\mathrm{m}}\right)$

Output: Majority voting (Test class)

\section{Fisher criterion based LDAs}

\section{Do}

1. Initialize the brain multiplex features.

2. Define class scatters and within class scatter using (6) and (7).

3. Fisher separation criterion is computed using (5).

4. From the original feature structure, $x$ features which are to be selected are initialized as (9).

5. Fisher separation criterion for these $x$ features is calculated using (10).

6. While $\mathrm{q}$ features are chosen Do

7. Select the $(q+1)^{\text {th }}$ feature that boosts the fisher criterion as (12).

8. The Fisher separation improvement value is calculated between the candidate feature and the last selected feature using (13).

9. The final feature selection criterion (14) consists of two functions, one is the Fisher separation criterion evaluating the discriminant characteristic of features and the other is the correlation term which measures the redundancy of features.

10 .

\section{End while}

\section{Correlational Analysis using DNN}

\section{Do}

11. Multiplex feature vectors are given as input to the DNN.

12. Update the weight and bias values of each hidden layer.

13. The activation function which is the output of the $y^{\text {th }}$ hidden layer is calculated using (18).

14. Activation function of the output layer is calculated using (19).

15. From the actual output, the error function is calculated using (20).

16. If the error of DNN structure is minimum DNN is considered as final trained structure.

17. Else Steps 2-6 are continued.

\section{End if}

19. With the Multiplex feature vectors, the trained DNN is tested and the predicted label is given as output.

\section{Majority voting}

20. Highly frequent class labels are obtained from two blocks with majority voting.

\section{RESULT AND DISCUSSION}

In this part, we discuss the result obtained from the suggested method. For implementing the proposed technique, we have used the Mat lab version (7.12). This proposed technique is done in the windows machine having an Intel Core i5 processor with speed $1.6 \mathrm{GHz}$ and $4 \mathrm{~GB}$ RAM. The proposed system has been tested on the data set available on the web. We have utilized the size of the image "512×512" which images are publicly available. 


\section{A. Evaluation Of Data}

Data from the Alzheimer's disease neuroimaging initiative (ADNI) database (adni.loni.usc.edu) are obtained in this research work. ADNI was launched as a public-private partnership in 2003 led by Michael W. Weiner, MD, Principal Investigator. ADNI's primary objective was to check how continuous magnetic resonance imaging (MRI), positron emission tomography (PET), other biological markers, and medical and neuropsychological evaluation can be used to assess the development of mild cognitive impairment (MCI) and early Alzheimer's disease (AD). 84 subjects, including $42 \mathrm{eMCI}$ and $42 \mathrm{AD}$, are used from this ADNI list.

\section{B. Performance Metrics}

We need numerous assessment metric values to be calculated to investigate our planned discriminative and correlational analysis. The metric values are found supported True Positive (TP), True Negative (TN), False Positive (FP) and False Negative (FN) with the choice of segmentation and grading. The quality of our planned work is analyzed by three metrics like Accuracy, Sensitivity, and Specificity.

\section{Experimental Analysis}

The performance of the proposed Correlational and discriminative is analyzed with the help of sensitivity, specificity, and accuracy. These are the most important performance parameters. The adequacy of the proposed technique is demonstrated by performing a comparison between the matching results of the proposed method with other approaches such as ensemble SVM paired classifier using ECFS and ensemble LDA and CCA SVM paired classifier. In this work, LDA for correlational analysis and optimized DNN for discriminative analysis are suggested. The result portion is split into two phases such as the right hemisphere and left hemisphere. We first consider the improvement of the right hemisphere.

Table. 1 shows the right hemisphere classification evaluations for our proposed method and existing methods. The accuracy of E-SVM -ECFS is 63.16 and E-LDA \& CCA -SVM achieves 72.62 and our proposed method 83.27. An E-SVM -ECFS obtains 67.32 sensitivity, 78.57 achieved by E-LDA \& CCA -SVM and 87.91 is for our proposed method fisher criterion-based LDA and optimized DNN. The specificity of our off methods achieves 67.76 and 71.43. Our proposed method obtains the specificity of 79.87 . The off methods achieve original features but that is low compared with our proposed method. Compared with other existing techniques our proposed technique achieves good accuracy 83.27, 87.91 sensitivity and specificity i.e. 79.87. The graphical representation of right hemisphere Accuracy, sensitivity and specificity are shown in fig.4,5 and6.
Table.1 Right hemisphere Classification valuation for Various Techniques

\begin{tabular}{|l|c|c|c|}
\hline \multicolumn{1}{|c|}{ Techniques } & Accuracy & Sensitivity & Specificity \\
\hline $\begin{array}{l}\text { E- SVM paired } \\
\text { Classifiers using } \\
\text { ECFS }\end{array}$ & 63.16 & 67.32 & 67.76 \\
\hline $\begin{array}{l}\text { E-LDA \& CCA-SVM } \\
\text { paired Classifier }\end{array}$ & 72.62 & 78.57 & 71.43 \\
\hline $\begin{array}{l}\text { Fisher Criterion } \\
\text { based LDA \& DNN }\end{array}$ & 83.27 & 87.91 & 79.87 \\
\hline
\end{tabular}

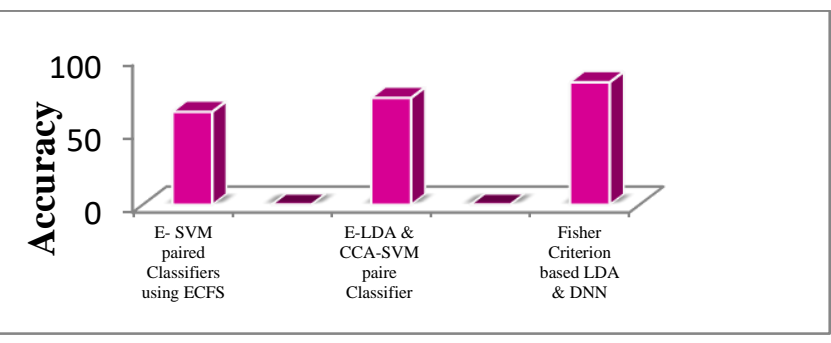

Fig.4 Graphical representation of right hemisphere Accuracy

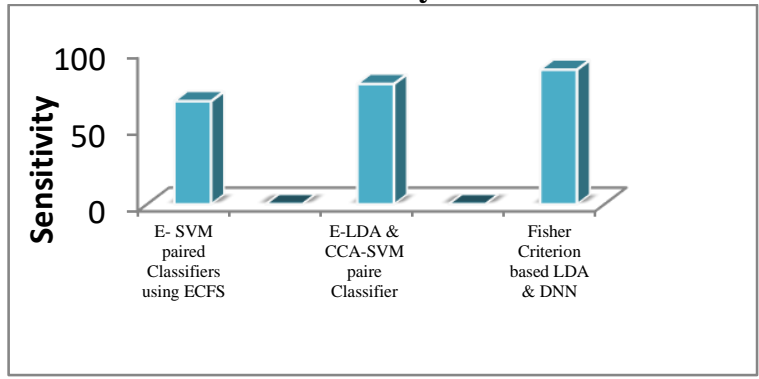

Fig.5 Graphical representation of right hemisphere sensitivity

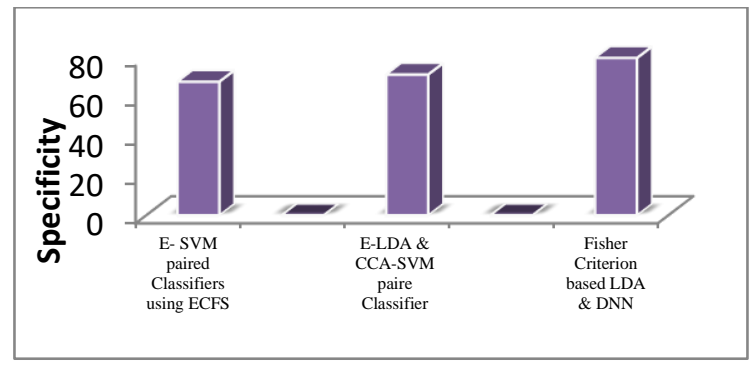

Fig.6 Graphical representation of right hemisphere specificity

Table.2 demonstrates the left hemisphere classification evaluations for our presented method and off methods. The accuracy of the proposed method is 89.72 and E-LDA \& CCA -SVM reaches 80.95 and 76.29 reaches E- SVM paired Classifiers using ECFS. The Sensitivity of existing method achieves 73.85, 83.33 and the Proposed method achieves sensitivity as 87.53. An Ensemble - SVM paired Classifiers using ECFS obtains 78.55 as specificity, 80.95 is achieved by the E-LDA \& CCA-SVM paired Classifier and 86.33 is for our proposed method. 
Table.2 Left hemisphere Classification Evaluation for Various Techniques.

\begin{tabular}{|l|l|l|l|}
\hline Techniques & Accuracy & Sensitivity & Specificity \\
\hline & & & \\
$\begin{array}{l}\text { E- SVM paired Classifiers } \\
\text { using ECFS }\end{array}$ & 76.29 & 73.85 & 78.55 \\
\hline & & & \\
$\begin{array}{l}\text { E-LDA \& CCA-SVM paired } \\
\text { Classifier }\end{array}$ & 80.95 & 83.33 & 80.95 \\
\hline $\begin{array}{l}\text { Fisher Criterion based LDA } \\
\text { \& DNN }\end{array}$ & & & \\
& & & \\
& & & \\
\end{tabular}

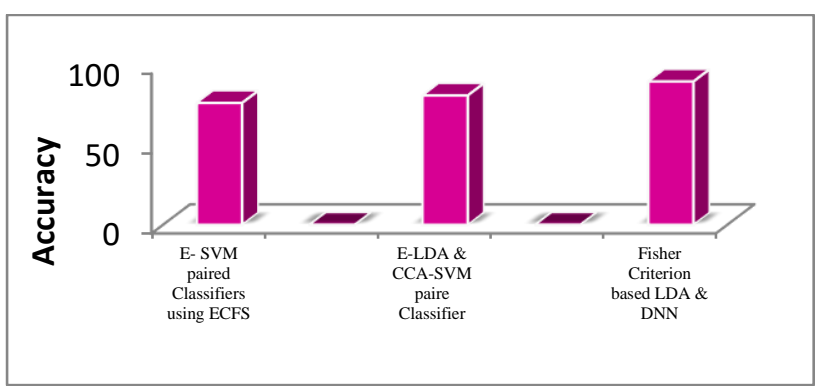

Fig.7 Graphical representation of left hemisphere accuracy

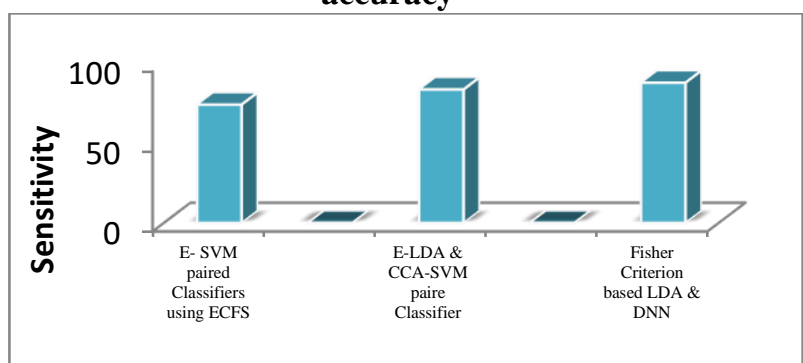

Fig.8 Graphical representation of left hemisphere sensitivity

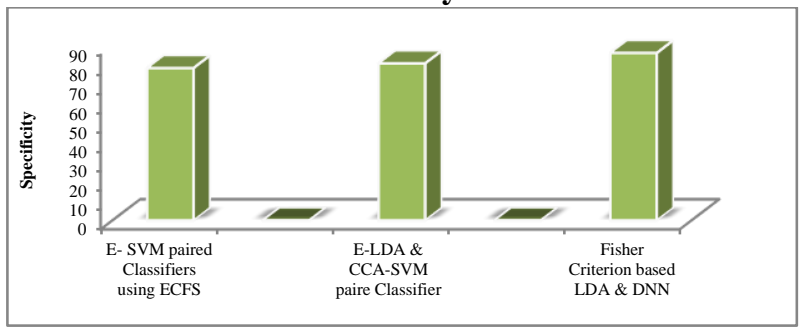

Fig.9 Graphical representation of left hemisphere specificity

Linear Discriminant Analysis (LDA) accomplishing the most astounding precision utilizing correlational information. These restricting outcomes may show that the beginning period of dementia influences the sides of the equator in unmistakable ways. While likewise delivering unrivaled outcomes with the proposed technique, it was additionally seen that blending both correlational and discriminative strategies altogether improved results for information on the correct side of the equator, when contrasted with any of the free discriminative strategies. The proposed procedure further enhanced the precision. From the above tabulation and graphical representation, we are known that the proposed method is better compared with other existing techniques.

\section{CONCLUSION}

In this work, to upgrade the correlational square, we exhibited an improved classifier additionally, for improving the presentation of the discriminative block, an optimized LDA is to be proposed. An enhanced LDA and its variations to distinguish brain characteristics include that discriminate between two brain states. The target of the proposed feature selection strategy is to some degree predictable with the LDA (both pursue the Fisher standard) and hence the combination of the proposed feature selection technique and LDA is relied upon to accomplish higher arrangement accuracy. For the correlational investigation, Optimized Deep Neural Network (DNN) is proposed in this work. DNN incorporates two stages: pre-preparing and tweaking stages in its parameter learning. The preparation procedure is accelerating with the guide of the LDA optimized algorithm. We demonstrated tabulations and graphical representations for the classification of the right and left hemispheres. Different evaluation metrics values to be calculated to analyze our proposed discriminative and correlational analysis. Here we use three evaluation metrics such as accuracy, sensitivity, and specificity. Based on the calculation metrics, we have known that our proposed method is outperformed compare with other off methods. In our future work, we will investigate multi-complex learning techniques for settling cerebrum views, which will inevitably deliver increasingly strong grouping results to anomalies in both unique and mapped spaces and we will coordinate practical and dispersion organizes in our multiplex structure to investigate how the connection between multimodal connectomic perspectives is modified with dementia beginning

\section{REFERENCES}

1. Jeon, H., Park, S., Choi, J. and Lim, Y., 2018, July. Ontology-based Dementia Care Support System. In 2018 40th Annual International Conference of the IEEE Engineering in Medicine and Biology Society (EMBC) (pp. 3318-3321). IEEE.

2. Jor'dan, A.J., McCarten, J.R., Rottunda, S., Stoffregen, T.A., Manor, B. and Wade, M.G., 2015. Dementia alters standing postura adaptation during a visual search task in older adult men. Neuroscience Letters, 593, pp.101-106.

3. Zhou, H., Jiang, J., Wu, P., Guo, Q. and Zuo, C., 2018, July. The disrupted network topology in patients with Lewy bodies dementia compared to Alzheimer's disease, Parkinson's disease dementia, and Health Control. In 2018 40th Annual International Conference of the IEEE Engineering in Medicine and Biology Society (EMBC) (pp. 1899-1902). IEEE.

4. Linz, N., Tröger, J., Alexandersson, J., Wolters, M., König, A. and Robert, P., 2017, November. Predicting dementia screening and staging scores from semantic verbal fluency performance. In 2017 IEEE International Conference on Data Mining Workshops (ICDMW) (pp. 719-728). IEEE.

5. Anuradha, G., Jamal, N. and Rafiammal, S., 2017, September. Detection of dementia in EEG signal using dominant frequency analysis. In the 2017 IEEE International Conference on Power, Control, Signals and Instrumentation Engineering (ICPCSI) (pp. 710714). IEEE. 
6. Al-Qazzaz, N.K., Ali, S., Islam, M.S., Ahmad, S.A. and Escudero, J., 2016, December. EEG markers for early detection and characterization of vascular dementia during working memory tasks. In 2016 IEEE EMBS Conference on Biomedical Engineering and Sciences (IECBES) (pp. 347-351). IEEE.

7. Isik, A.T., Kocyigit, S.E., Smith, L., Aydin, A.E., and Soysal, P., 2019. A comparison of the prevalence of orthostatic hypotension between older patients with Alzheimer's Disease, Lewy body dementia, and without dementia. Experimental gerontology, 124, p.110628.

8. Hong, C.T., Chan, L., Wu, D., Chen, W.T. and Chien, L.N., 2019. Antiparkinsonism anticholinergics increase dementia risk in patients with Parkinson's disease. Parkinsonism \& Related Disorders.

9. Raeper, Rory, Anna Lisowska, and Islem Rekik, "Cooperative correlational and discriminative ensemble classifier learning for early dementia diagnosis using morphological brain multiplexes," IEEE Access, Vol. 6, pp. 43830-43839, 2018.

10. Kawanishi, K., Kawanaka, H., Takase, H. and Tsuruoka, S., 2017, September. A study on dementia detection method with stroke data using anomaly detection. In 2017 6th International Conference on Informatics, Electronics and Vision \& 2017 7th International Symposium in Computational Medical and Health Technology (ICIEV-ISCMHT) (pp. 1-4). IEEE.

11. Chen, D., Jiang, J., Wu, P., Guo, Q. and Zuo, C., 2018, July. Module differences of glucose metabolic brain network among Alzheimer's disease, Parkinson's disease dementia, Lewy body dementia, and health control. In 2018 40th Annual International Conference of the IEEE Engineering in Medicine and Biology Society (EMBC) (pp. 2036-2039). IEEE.

12. Bharanidharan, N. and Rajaguru, H., 2018, December. Classification of Dementia Using Harmony Search Optimization Technique. In 2018 IEEE Region 10 Humanitarian Technology Conference (R10-HTC) (pp. 1-5). IEEE.

13. Kobayashi, T., Miyazaki, T. and Arai, K., 2018, October. Dementia Diagnosis System Using Social Media Agency Robot. In 2018 Eleventh International Conference on Mobile Computing and Ubiquitous Network (ICMU) (pp. 1-2). IEEE.

14. Grande, G., Triolo, F., Nuara, A., Welmer, A.K., Fratiglioni, L., and Vetrano, D.L., 2019. Measuring gait speed to better identify prodromal dementia. Experimental gerontology

15. Schrum, M., Park, C.H. and Howard, A., 2019, March. Humanoid Therapy Robot for Encouraging Exercise in Dementia Patients. In 2019 14th ACM/IEEE International Conference on Human-Robot Interaction (HRI) (pp. 564-565). IEEE.

\section{AUTHOR'S PROFILE}

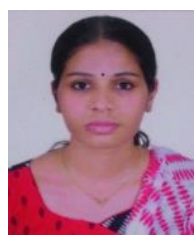

Mrs. Ambili A.V, is currently a full time student at Hindustan college of Arts and Science, Coimbatore. she has completed her MCA from IGNOU.her field of research interest is data mining.

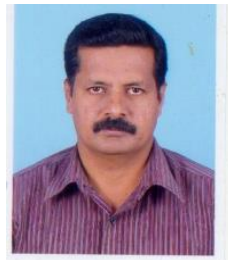

Dr. A. V. Senthil Kumar, obtained his Ph.D in Computer Science. He has to his credit 9 Book Chapters, 174 papers in International Journals, 4 papers in National Journals, 25 papers in International Conferences, 5 papers in National Conferences and edited five books in Data Mining, Mobile Computing, Fuzzy Expert Systems, Biometric Authentication and Web Mining (IGI Global, USA). $\quad \mathrm{He}$ is an Editorin-Chief for 4International Journals and Key Member forIndia, Machine Intelligence Research Lab (MIR Lab). He is an Editorial Board Member and Reviewer for various International Journals. He is also a Committee member for various International Conferences

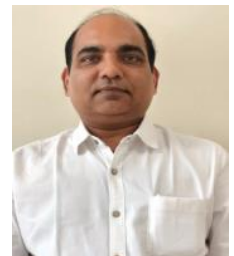

Dr. Amit Dutta, is a Director at AICTE HQ New Delhi and has been the Regional Director, Western Region, AICTE Mumbai for 2 years. He is also a team leader of Online Portal Development for One Lakh Fifty Thousand Students and 400 colleges for examination and affiliation system. He is working as a Senior Programmer in Department of Computer Science and Applications, Barkatullah University Bhopal. And also experienced as a coordinator for RUSA, AISHE(M.P) and AISHE(GOI) and Design and Development Of Portal to capture Gross Enrolment Ratio data for M.P.A result oriented Administrator, academician and scientific researcher with 19+ years of experience in strategizing, designing, modelling and developing secure framework and solutions for problems. Proven track record in Problem analysis, vulnerability assessment, design, development and implementation of safe secure Solution for all Administrative matters.He graduated with Msc. Maths and started study PGDCA.He got his ph.D in Mathematics \& Computer Science from Bhopal University and also specialization in Neural Networks. He has Published few books such as "C++ \& Data Mining Techniques",and has also Published Papers In International JournalsDr.Amit Dutta was Nominated for Attending Meeting by the Board of Governors at IIM Bodggaya,SambalpurHe was the Editorial Board Member in

- International Journal Of Scientific and Engineering IJSER,India

- International Journal Of Scientific Knowledge(Computing and Information Technology)

- International Journal Of Data Mining Techniques and Applications India 